\title{
Algunos apuntes sobre la evaluación de diccionarios
}

\author{
$M^{a}$ Teresa Fuentes Morán \\ Joaquín García Palacios \\ Jesús Torres del Rey \\ Universidad de Salamanca
}

Nos encontramos en un momento en el que los productos lexicográficos empiezan a experimentar cambios profundos. Cambian los formatos, lo que tiene importantes consecuencias en los mecanismos de transmisión de la información, cambian las bases documentales, los métodos de explotación de datos..., pero además, los cambios que se producen en las formas de acceso a la información de las que disponemos hoy en día tienen como consecuencia que los usuarios de los diccionarios se acerquen a ellos con otras expectativas o, de manera más o menos consciente, con otros tipos de necesidades. En este contexto, parece lícito preguntarse cuál es el papel que el diccionario desempeña y reconocer que las obras lexicográficas actuales van adquiriendo nuevas funciones, o cuando menos funciones distintas a las que se les asignaba tradicionalmente. Es cierto que estamos inmersos en lo que se ha dado en llamar sociedad de la información, que las posibilidades de acceso a la información son crecientes gracias al desarrollo de las nuevas tecnologías, y es esto precisamente lo que hace que los receptores de esa información se vean en la acuciante necesidad de valorarla adecuadamente, de medir su fiabilidad. Los diccionarios, por naturaleza ricos portadores de determinados y variados tipos de datos, deben ser ahora susceptibles de nuevas formas de análisis que permitan a sus usuarios determinar el grado de confianza que en ellos depositan. Y esto con la conciencia de que su papel va cambiando en la actualidad y de que nos enfrentamos a un tipo de obra que con frecuencia se sitúa como eje del engranaje informativo del que disponen sus usuarios. 
No pretendemos evaluar o valorar aquí obras del pasado -aun del más próximo- con criterios que les son ajenos, pero sí constatamos la necesidad de desarrollar criterios objetivos que permitan delimitar los parámetros de medición de calidad de las obras lexicográficas. Y esto respetando sus propios principios, pero desde el punto de vista de su adecuación al estado actual del conocimiento, a las funciones que orientan a sus creadores y, muy especialmente, al papel que sus destinatarios actuales les otorgan. Pero siempre desde la perspectiva del usuario, pues es ésta la que puede proporcionarnos información certera sobre la utilidad de la obra.

Entre los años ochenta y los noventa se presentaron interesantes trabajos que aportaban algunos criterios pertinentes para la evaluación de diccionarios. No obstante, hay varias razones que ponen de manifiesto la necesidad de proseguir la investigación en estos aspectos. Por un lado, el hecho de que algunos de estos criterios hayan caído en el olvido, por lo menos en lo que se refleja aún en las reseñas de diccionarios; por otro lado, y más importante aún, las nuevas generaciones de diccionarios deben responder a otras nuevas expectativas, encuentran distintos tipos de perfiles de usuarios y se elaboran con distintos recursos textuales, lo que debe llevarnos a establecer nuevos parámetros para el juicio.

El diccionario es una obra compleja que puede ser observada desde muy distintos puntos de vista, y si bien es cierto que se están dando cambios en el papel que el diccionario desempeña, también debemos señalar la dificultad que todavía existe para delimitar estos cambios en parámetros concretos (cf. Humbley 1993: 63). Aun hoy las reseñas de diccionarios presentan como argumento principal listas de unidades léxicas que faltan o sobran, y propuestas de clases de componentes adecuados al tipo de diccionario de que se trate:

En la mayoría de los casos, los críticos de diccionarios lo que hacen es señalar aquello que, a su juicio, falta y censurar lo que, a su parecer, sobra (Salvador 2002: 237).

Algunos autores han tratado de establecer métodos más objetivos para la valoración de diccionarios. Si nos acercamos a las propuestas 
tradicionales, podemos ver, por ejemplo, lo que Haensch y Omeñaca (2004: 329-334) presentan en el siguiente esquema que puede servir "como una especie de guía práctica para los autores de reseñas de diccionarios, vocabularios, glosarios y otros inventarios lexicográficos" (329):

1. Datos bibliográficos

2. Caracterización tipológica

3. Evaluación formal (cuantitativa y cualitativa del diccionario)

-Descripción de la macroestructura del diccionario

-Análisis de la microestructura del diccionario

4. Evaluación del contenido

-Selección de entradas

-Explicación de las unidades léxicas

5. Ilustraciones

6. Tipografía

La propuesta de Humbley para los diccionarios de especialidad se organiza a partir de los siguientes criterios:

Accesibilidad:

¿Cómo encontrar el diccionario? Referencias, compra, consulta, selección

Autoridad:

Autor, aval, referencias

Tema (o campo tratado):

¿Cuáles son los campos tratados? Campo restringido, campo amplio, selección

Cantidad de lenguas: Unilingüe, bilingüe, plurilingüe

Estructura de Definición, remisiones, informaciones

los artículos no entradas del diccionario: lingüísticas, sinonimia, polisemia, contextos, nota técnica

Entre los parámetros más recurrentes en estas propuestas se encuentran la caracterización tipológica del diccionario, su adecuación a las funciones lexicográficas que se le atribuyen y al usuario prototípico al que se destina la obra (cf. Gelpí 1995 y 1997), y, especialmente, la evaluación externa de la obra desde el punto de vista de sus componentes.

Desde algunos ámbitos se reclama la crítica de diccionarios como componente imprescindible de la lexicografía teórica o metalexicografía. Una muy coherente propuesta de sistematizar la crítica de dicciona- 
rios, expuesta por R. Werner en diversas conferencias y recogida en Haensch y Omeñaca (2004: 335-336), se basa en tres pilares fundamentales:

a) La descripción de las características de la obra lexicográfica que debe someterse a examen.

b) La delimitación del marco de referencia para la evaluación de las características observadas.

c) El establecimiento de los diferentes procedimientos a través de los cuales se puede examinar la obra lexicográfica.

Así, frente a la crítica tantas veces tachada de impresionista de algunos reseñadores, se utilizan otros métodos de evaluación que tratan de valorar el rendimiento de la obra. Ante la imposibilidad de llevar a cabo un estudio integral, se van establecido parámetros para emprender análisis estadísticamente representativos, que por lo general se limitan a su evaluación cuantitativa (cf. Rettig 1985 y Fuentes Morán 1997). La adecuación del contenido se analiza con frecuencia mediante encuestas a usuarios. Un método de análisis que logró una aceptación muy generalizada es el de la consulta de la obra lexicográfica a partir de situaciones de uso reales o en las que se emulan situaciones reales; estas consultas las puede hacer el propio reseñador o evaluador -con los condicionamientos que esto supone- o pueden hacerlas usuarios que son observados por el evaluador o bien redactan informes o protocolos de uso, que se someten posteriormente a análisis. Son estos sólo ejemplos de las más importantes propuestas metodológicas; es muy probable que investigaciones posteriores modelen los métodos de evaluación empleados hasta ahora.

Desde nuestro punto de vista, es necesario facilitar al usuario métodos más abarcadores que le faciliten el conocimiento de la obra y que estén basados en parámetros más generales y útiles para un amplio abanico de diccionarios. A su vez, el especialista o el reseñador podrá emplear los mismos métodos, que él podrá seguir con un conocimiento que le permite realizar análisis más profundos y valoraciones más fundamentadas. Estos juicios serán entonces de mayor utilidad para los receptores ñtanto si se trata de usuarios como de especialistas en algún ámbito de la lexicografía. 
La propuesta a la que queremos llegar, y que aquí exponemos sólo muy parcialmente, la fundamentamos en los dos principios siguientes:

1. ¿Qué objeto se evalúa?

Como ya hemos señalado, el diccionario, considerado como herramienta, ha pasado a ser uno de los elementos -importante, eso sí- del engranaje informativo con el que cuenta un usuario para resolver las cuestiones que se le plantean sobre objetos lingüísticos. Junto con el diccionario, el usuario actual está cada vez más acostumbrado a utilizar otras fuentes de información, generalmente no específicas, de las que va extrayendo, de forma más o menos costosa y más o menos afortunada, la información que precisa. Por ello, es cada vez más importante valorar la capacidad del diccionario de formar parte de ese engranaje del que hablamos, de explicitar la información lingüística implícita en otras fuentes o de vehicularla.

2. ¿Quién evalúa?, ¿con qué perspectiva?

Sobre este aspecto parece incuestionable que la fiabilidad de la evaluación depende en gran parte del grado de conocimiento que del objeto, en sus distintos aspectos, que tenga el evaluador. Pero lo que nosotros planteamos aquí no es la evaluación en este sentido, sino que tratamos de encontrar mecanismos para que el usuario, sea éste quien sea, pueda valorar adecuadamente la obra que tiene en sus manos. Por otro lado, parece lógico considerar que es la perspectiva del usuario el punto de referencia válido para la evaluación de la obra lexicográfica, puesto que a él va dirigida primariamente. Esto no quiere decir que se invalide la valoración del especialista o del lingüista, sino que éstos deberían en todo caso emitir su juicio adoptando el punto de vista del destinatario tipo del diccionario. Es un hecho que el evaluador, el reseñador, el especialista en lexicografía o el usuario tienen formas distintas de acercarse al diccionario, y probablemente distintas expectativas. Pero, al final, lo que hay que medir es su operatividad, basada en usos reales o posibles, y ésta no en términos absolutos, sino en términos relativos para evitar el hecho que se produce con frecuencia que consiste en achacarle una imperfección a un diccionario que lo único que pone de manifiesto es la ignorancia del reseñador: 
Hay que hacer calas bien orientadas, comparativas en la medida de lo posible, y no pedirle más de lo que explicita y confesadamente ofrezca. No han faltado, en el caso del $D E A$, quienes echen de menos, en sus comentarios, testimonios del español de América, sin caer en la cuenta de que se trata de un diccionario descriptivo limitado a un tiempo establecido y a un espacio geográfico limitado (Salvador 2002: 236).

Así, y sin perder de vista el carácter de herramienta que otorgamos al diccionario, propondremos algunos de los muchos parámetros que pueden servir para evaluar estas fuentes de referencia. Estos elementos de juicio pueden articularse en tres ejes en los que consideramos que se puede organizar toda evaluación de este tipo de obras.

a) Cantidad de datos lexicográficos

b) Calidad de los datos lexicográficos

c) \#Usabilidad del diccionario.

Pero estos tres ejes convergen en cada una de las consultas que pueden hacerse en el diccionario y, por lo tanto, deben también converger en los elementos que se empleen para su evaluación. De poco servirá un diccionario en el que no esté bien resuelto cada uno de estos puntos; así, en lugar de tratar de establecer los componentes de cada uno de estos tres ejes, lo que parcelaría el análisis en compartimentos inexistentes en el uso real, trataremos de plantear cuestiones desde una perspectiva más abarcadora.

Presentaremos algunas de las cuestiones en las que puede formularse una guía para la evaluación y que se pueden resumir en la siguiente: ¿Se puede con el diccionario completar o corroborar correcta y fluidamente la información obtenida mediante otras fuentes de información?

En realidad, la consulta genuina del diccionario siempre parte de información externa. Al diccionario se llega, por ejemplo, ante una duda sobre una unidad léxica encontrada en un texto o que nos viene a la mente. Esto es también así cuando el diccionario se utiliza para alguna de las funciones que llamamos activas, puesto que su consulta debe siempre partir de una unidad léxica conocida. 
Estos aspectos, que se concretan en cada diccionario y en cada tipo de consulta, pueden verse formulados, especialmente, en las siguientes preguntas, que serán más o menos pertinentes en relación con cada caso determinado:

I: ¿Ofrece el diccionario suficientes datos?

II: ¿Se puede encontrar fácil y rápidamente la información que se busca y que el diccionario contiene?

III. ¿Son los datos inteligibles?

IV. ¿Permite el diccionario valorar su fiabilidad?

Comentemos brevemente algunas cuestiones acerca de cada una de ellas.

I. ¿Ofrece el diccionario suficientes datos?

Los datos sobre el número de entradas no son fácilmente delimitables porque no son fácilmente objetivables. Los primeros elementos de la descripción de un diccionario suelen incluir el número de entradas que éste recoge, y en algunos casos se habla además del número de acepciones o de definiciones, e incluso del número de unidades pluriverbales. Evidentemente, este dato resulta relevante puesto que da una idea aproximada del tamaño del mismo, lo que muchos consideran un primer dato objetivo, que sin embargo merece cautela. Así, por ejemplo, una unidad puede ser tratada como unidad polisémica, o puede ser presentada en dos o más artículos, con la consideración de unidades homónimas; la forma femenina de determinadas unidades puede ser lematizada con el masculino o por separado. En ambos casos, de una u otra decisión resulta un número diferente de elementos que solemos llamar "lemas", sin que pueda afirmarse que haya diferencia en cuanto al contenido.

Por lo que respecta al programa informativo del artículo lexicográfico, queremos señalar aquí dos aspectos que consideramos de especial relevancia:

- La importancia de no identificar cada elemento formal del diccionario con una clase de información. Es decir, dada la necesaria redundancia del texto lexicográfico, los tipos de información que contiene el diccionario no se identifican en relación simétrica con 
las partes formales del texto lexicográfico. Esta identificación es frecuente cuando se trata de establecer las clases de información que contiene un diccionario y lleva por ejemplo a considerar lo que muchos llaman marca gramatical como el único elemento portador de información gramatical explícita en el artículo lexicográfico.

- En determinados tipos de consultas, frecuentes hoy en día, se hacen necesarios otros tipos de información considerada accesoria hace años, pero sin la cual ahora no se garantiza el funcionamiento de ese engranaje informativo del que hablamos. Así, según el tipo de diccionario de que se trate pueden hacerse otras preguntas sobre los tipos de información, como pueden ser: ¿ofrece datos sobre el tipo de texto en el que puede encontrarse la unidad léxica en cuestión? (por ejemplo, texto especializado frente a texto no especializado), ¿ofrece datos sobre la proyección sintáctica de la unidad léxica en cuestión? o ¿resuelve dudas sobre las implicaciones pragmáticas del uso de esta unidad?

II. ¿Se puede encontrar fácil y rápidamente la información que se busca y que el diccionario contiene?

Para que se cumpla esta condición el diccionario debe presentar los datos adecuadamente organizados, es decir, estructurados en vías de acceso que no se interrumpan y con formatos flexibles que no hagan necesario verbalizar qué tipo de información se requiere. Los nuevos formatos de diccionarios hacen posibles -y a la vez imprescindiblesprocedimientos de jerarquización de los datos de acuerdo con los tipos de usuarios previstos; este es uno de los aspectos que requieren más profunda investigación en la lexicografía actual.

\section{III. ¿Son los datos inteligibles?}

Nos encontramos aquí frente a uno de los aspectos más relevantes en un diccionario con definiciones, pero también ante una de las que resultan más difíciles de evaluar con elementos objetivables, ya que depende directamente de mecanismos de comprensión del usuario, de sus procedimientos de adquisición del conocimiento, de sus hábitos heurísticos. Veamos algunos parámetros para valorar en este aspecto las definiciones. 
Independientemente del tipo formal de definición por la que se haya optado, podemos considerar que la definición lexicográfica se basa en una estructura tema/rema que (parcialmente) se corresponde con el binomio información conocida/información desconocida (es decir, en este caso, información que se pretende proporcionar y que en el diccionario, como en otros casos, puede reflejar distintas estructuras). La inteligibilidad de la definición puede medirse, especialmente, en los siguientes puntos:

a) Grado de dificultad de los elementos empleados en la definición y de las posibilidades del usuario de resolver ese grado de dificultad con el propio diccionario de manera ergonómica y satisfactoria.

b) Coherencia y adecuación en la organización de los elementos en las definiciones, teniendo en cuenta factores como los siguientes: ¿cómo percibe la sociedad un determinado concepto?, ¿cómo lo percibe un lingüista u otro tipo de especialista?, ¿qué tipo de percepción se pretende transmitir a través de los datos integrados en el artículo lexicográfico?

c) Coherencia entre los elementos microestructurales externos a la definición con la propia definición, aspecto que se mide en parámetros de redundancia y complementariedad -nunca de contradicción.

IV. ¿Permite el diccionario valorar su fiabilidad?

Este aspecto ha adquirido en los últimos años una relevancia especial en esa sociedad de la información de la que hablábamos más arriba y considerando la incesante y creciente publicación de diccionarios a la que asistimos actualmente.

a) ¿Se proporcionan datos sobre la autoría de la obra? La fiabilidad de un diccionario puede medirse en primer lugar por el prestigio de quien lo ha elaborado. Está claro, pero esto es aún más importante hoy en día. Por ejemplo, es importante saber en un diccionario de especialidad a qué escuela concreta pertenece el grupo redactor, en un diccionario bilingüe debe saberse en qué ámbito lingüístico se ha elaborado; en un diccionario general, cuáles son los presupuestos teóricos, por ejemplo lexicográficos, que lo sustentan. 
b) ¿Se proporcionan datos concretos sobre las fuentes utilizadas tanto para el vaciado de las unidades léxicas que forman parte de la macroestructura como para la obtención del resto de los datos que se proporcionan?, es decir, el origen de los datos. En este contexto es en el que debe obtenerse información sobre la actualidad de los datos. Para la elaboración de los diccionarios se usan hoy en día especialmente dos tipos de fuentes primarias: datos procedentes de otras fuentes lexicográficas y datos procedentes del vaciado de corpus; si bien la segunda fuente es la más prestigiada en la actualidad, aún deben desarrollarse mecanismos más certeros para elaboración y vaciado de corpus de referencia.

No queremos terminar esta breve exposición sin plantear aún algunas observaciones. La corroboración de los datos que proporciona el diccionario sólo puede hacerse mediante mecanismos externos; los datos del propio diccionario son sólo indicios de su fiabilidad; cuando mayor es el número de indicios encontrados y estos se presentan de manera más explícita, más fiable podemos considerar el diccionario. Pero no podemos establecer una relación directa entre los indicios y la calidad de la obra.

Indicios de calidad -y de fiabilidad porque reflejan los métodos de trabajo adoptados en el diccionario- son:

- Coherencia formal (por ejemplo, en marcadores estructurales).

- Indicaciones sobre el origen de los datos y sobre los sistemas de procesamiento fines lexicográficos.

- Indicaciones sobre la finalidad del diccionario y los destinatarios a los que se orienta.

- Toma explícita de postura frente a algunos aspectos (por ejemplo socioculturales).

- Indicios sobre la actualidad de los datos, etc.

Concluiremos con unas palabras de G. Salvador:

Lo cierto es que, pese a todo lo dicho, del diccionario puede hablar cualquiera, porque el diccionario es de todos los hablantes de la lengua cuyo léxico describa. Todos llevamos un ejemplar en el cerebro, ya lo dijo Saussure, en la tercera circunvolución frontal izquierda, pe- 
ro es siempre un ejemplar parcial, mutilado, incompleto, a veces borroso o confuso en algunas entradas, y lo que buscamos en el que está escrito, en el del grueso libro imprescindible, es ajustar el de nuestra mente, completarlo, aclararlo, enriquecerlo y, por supuesto, discutir el de fuera, ponerlo en tela de juicio, que para eso tenemos el testimonio del nuestro y su estructura, y la queremos trasladar al otro para mejorarlo, para hacerlo más a nuestro modo, porque de lo propio, se mire como se mire, se fía uno siempre mucho más (Salvador 2003: 239).

Pero aprender a valorar las obras lexicográficas con criterio enseña también utilizar críticamente los diccionarios.

\section{BIBLIOGRAFÍA}

CARrizo, G. (2000): Manual de fuentes de información, Zaragoza, Confederación Española de Gremios y asociaciones de Libreros.

Cordón García, J. A., J. López Lucas y J. R. Vaquero Pulido (2001): Manual de investigación bibliográfica y documental, Madrid, Pirámide.

Fuentes MORÁN, Mª T. (1993): "Wörterbuchkritik im spanischen Sprachraum. Rezensionen in fünf Fachzeitschriften 1975-1990", Lexicographica, 9, pp. 36-57.

Fuentes MORÁN, Ma T. (1997): Gramática en la lexicografía bilingüe, Tübingen, Max Niemeyer.

GelPí ARroYo, C. (1995): Els diccionaris bilingües: mesures d'avaluació. Primera proposta, Tesis de licenciatura, Barcelona, IULA.

Gelpí ARroyo, C. (1997): Mesures d'avaluació lexicogràfica de diccionaris bilingües, Tesis doctoral, Barcelona, IULA.

Humbley, J. (1993): "La evaluación de un diccionario especializado", Terminometro, $13-14$, pp. 62-63.

HAENSCH, G. (1985-1986): "La crítica de diccionarios", Boletín de la Academia Norteamericana de la Lengua Española, 6-7, pp. 53-62.

HAENSCH, G. y C. OMeÑACA (2004): "La crítica de diccionarios", en G. Haensch y C. Omeñaca, Los diccionarios del español en el umbral del siglo XXI, Salamanca, Universidad de Salamanca, pp. 329-337.

JEHLE, G. (1990): Das englische und französische Lernerwörtebuch in der Rezension. Theorie und Praxis der Wörterbuchkritik, Tübingen, Max Niemeyer. 
Martín Vega, A. (1995): Fuentes de información general, Gijón, Trea. RetTig, W. (1985): "Die zweisprachige Lexikographie FranzösischDeutsch, Deutsch-Französisch. Stand, Probleme, Aufgaben", Lexicographica, 1, pp. 83-124.

RIPFEL, M. (1989): Wörterbuchkritik. Eine empirische Analyse von Wörtebuchrezensionen, Tübingen, Max Niemeyer.

SAlvador, G. (2003): "La crítica de diccionarios", en P. Álvarez de Miranda y J. Polo (eds.), Lengua y diccionarios. Estudios ofrecidos a Manuel Seco, Madrid, Arco Libros, pp. 235-239.

Segoviano, C. (1984): "Criterios para la valoración de un diccionario bilingüe", en C. Segoviano y J. M. Navarro (eds.), Spanien und Lateinamerika. Beiträge zur Sprache, Literatur und Kultur. Homenaje a Anton e Inge Bemmerlein, Stuttgart, DSV, pp. 474-495.

Torres Ramírez, I. (1998): Fuentes de información: estudios teóricoprácticos, Madrid, Síntesis. 